\title{
Comparison of Miscibility and Morphology in Two Ternary Blend Systems: Effects of Binary Interactions
}

\author{
Li Ling Chang, Ea-Mor Woo, ${ }^{\dagger}$ and Hung-Ling LiU \\ Department of Chemical Engineering, National Cheng Kung University, Tainan, 701, Taiwan
}

(Received June 14, 2004; Accepted August 23, 2004; Published November 15, 2004)

\begin{abstract}
Experimental and theoretical studies on two ternary blend systems comprised of polystyrene (PS), isotactic polystyrene (iPS) and poly(cyclohexy methacrylate) (PCHMA) or poly(2,6-dimethyl-p-phenylene oxide) (PPO) are described. The differences in phase behavior and morphology of PS/iPS/PCHMA and PS/iPS/PPO ternary blends were investigated and compared by using differential scanning calorimeter (DSC), polarized-light optical microscopy (POM), and scanning electron microscopy (SEM). These two ternary blend systems were found to be completely miscible within the entire composition range at ambient temperature. However, cloud-point transition and LCST behavior was observed for the PS/iPS/PCHMA blend system, but not for the PS/iPS/PPO blend system. Phase diagrams for ternary systems of PS/iPS/PCHMA $v s$. PS/iPS/PPO at elevated temperatures of cloud points were constructed with the experimental data. Theoretical calculations were found to agree well with the data. Interaction parameters in the PS/iPS/PCHMA $v s$. PS/iPS/PPO ternary blends were evaluated to assess possible $\Delta \chi$ among all the binary pairs that constitute these two ternary blends. The melting point depression was used to investigate interactions between pairs of PS/PCHMA vs. PS/PPO, and it was found that asymmetry in the binary interactions led to more ternary phase instability, which was manifested as lowering the LCST and/or cloud points. [DOI 10.1295/polymj.36.909]

KEY WORDS Ternary Polymer Blend / Phase Behavior / LCST / PPO / PS / PCHMA / iPS /
\end{abstract}

Truly miscible ternary blends composed of three polymers different in properties may offer a unique opportunity to develop a new polymer material with flexible combination from three constituents. An approach via ternary systems offers more flexibility and better property balancing than what can be achieved by binary blends. Recent advance in studies on ternary polymer blends have resulted in a few more discoveries on miscible ternary blends. The miscibility of ternary polymer blends consisting of three miscible binary pairs has also been studied. ${ }^{1-6}$ The fact that each binary pair is miscible does not guarantee that all ternary compositions will be. Depending on the interaction parameters of the three binary pairs, the ternary blend system may be completely miscible $3,7,8$ or may show an immiscible loop. ${ }^{9,10}$ Asymmetry in binary interaction parameters, the so-called $\Delta \chi$ effect, usually promotes phase separation and enlarges the immiscibility region..$^{9-11}$ Kwei et al. ${ }^{12}$ have reported ternary polymer blends of poly(methyl methacrylate) (PMMA), poly(ethyl methacrylate) (PEMA), and poly(vinylidene fluoride) (PVDF), in which the binary pair of PMMA and PEMA is immiscible; however, the addition of PVDF to the immiscible binary blend PMMA/PEMA can form a miscible ternary blend. Nishi et al. ${ }^{13}$ have investigated the phase behavior of ternary polymer blends and analyzed their phase diagrams according to the Flory-Huggins-Scott theory. Ternary blends of PMMA, poly(epichlorohydrin), and PEO are miscible, where all three binary pairs are miscible. ${ }^{14}$

Lacking any special functional groups in the chain structure, polystyrene (PS) is immiscible with most long-chain thermoplastic polymers. Blends involving one styrenic polymer are already rarely miscible; hence, blends comprising two styrenic polymers (different structures) are expected to be even less likely to be miscible. But three blend systems comprising PS are known to be miscible. One is the blend system of PS with poly(2,6-dimethyl-p-phenylene oxide) (PPO), which is one of most studied polyblend, and its miscibility has been demonstrated since early time. ${ }^{15-17}$ Second example of miscible blends comprising PS and an ether-containing polymer is given by the classical aPS/poly(vinyl methyl ether) (aPS/ PVME) system. ${ }^{18-20}$ Another notable example of miscible blends comprising two styrenic polymers (with different tacticity) is given by the PS/isotactic polystyrene (PS/iPS) system ${ }^{21,22}$ PS and poly(cyclohexyl methacrylate) (PCHMA) have been shown to be miscible at room temperature under certain condition. For relatively low molecular weights of PS (e.g., $M_{\mathrm{n}}=$ $90,000 \mathrm{~g} / \mathrm{mol}$ or lower), the PS/PCHMA blend system is miscible; for higher molecular weights of PS, the blend becomes immiscible. ${ }^{23,24}$ The blend exhibits lower critical solution temperature (LCST) behavior, 
with a miscibility region dependent on the molecular weight of the components. The structure of PS was replaced with regular tacticity, i.e., PS was replaced by isotactic polystyrene (iPS). Mixtures of all compositions involving semi-crystalline iPS and amorphous PCHMA are miscible and exist a LCST behavior. ${ }^{25,26}$ The tacticity of PS does not seem to adversely influence the miscibility in blends of iPS/PCHMA. The phase behavior of the iPS/PCHMA blend system was deemed more stable than that of PS/PCHMA at elevated temperatures. This result is corroborated by the LCST curves.

Several methods ${ }^{27-29}$ have been used to evaluate $B$ values of miscible polymer pairs. Among them, the melting point depression method has been used for blends where one of the components is semi-crystalline. The melting point depression of a semi-crystalline polymer in a mixture is due to a decrease of the chemical potential of the amorphous phase of the blend.

Most studies of ternary A/B/C systems reported in the literature ${ }^{1-6}$ deal with the addition of a miscible polymer $\mathrm{C}$ to a miscible $\mathrm{A} / \mathrm{B}$ pair, with $\mathrm{A} / \mathrm{C}$ and $\mathrm{B} / \mathrm{C}$ pairs being miscible. In PS/iPS/PPO and PS/ iPS/PCHMA ternary blend systems, each binary pair forms miscible blends because of favorable polymerpolymer interactions. For example, miscibility of PS/ iPS has been confirmed by numerous studies. ${ }^{21,22}$ The four following binary systems, PS/PCHMA, iPS/ PCHMA, PS/PPO, and iPS/PPO, are also miscible. In this study, we further considered ternary PS/iPS/ PCHMA and PS/iPS/PPO blends to investigate the possibility of rendering two miscible polystyrene homopolymers by adding PCHMA and PPO, respectively. In addition, we attempted to evaluate the thermodynamic interaction energy densities of all blends and calculate the ternary phase boundary and compare to the experimental phase diagram.

\section{EXPERIMENTAL}

\section{Materials and Sample Preparation}

Tactic polystyrenes were available from specialtypolymer suppliers and thus in-house synthesis was not necessary. A research-grade atactic polystyrene (aPS, or simply PS) was obtained from Chi-Mei, Inc. (Taiwan), with $\bar{M}_{\mathrm{w}}=192,000-236,000 \mathrm{~g} / \mathrm{mol}$, polydispersity index $(\mathrm{PI})=2.5-3.1$, and $T_{\mathrm{g}}=85^{\circ} \mathrm{C}$. Isotactic polystyrene (iPS) was purchased from Scientific Polymers Products ( $\mathrm{SP}^{2}$, Inc. USA), with a $T_{\mathrm{g}}$ of $95^{\circ} \mathrm{C}, \bar{M}_{\mathrm{w}}=400,000 \mathrm{~g} / \mathrm{mol}$. Poly (cyclohexyl methacrylate) (PCHMA) was purchased from Aldrich, Inc. (USA), with an approximate $\bar{M}_{\mathrm{w}}=65,000 \mathrm{~g} /$ mol (gel permeation chromatograph, GPC) and a $T_{\mathrm{g}}$ of $110^{\circ} \mathrm{C}$. Poly(2,6-dimethyl-p-phenylene oxide) (PPO) was purchased from a specialty-polymers sup- plier (Polysciences, USA), with an approximate $\bar{M}_{\mathrm{w}}=$ $50,000 \mathrm{~g} / \mathrm{mol}(\mathrm{GPC})$, polydispersity index $(\mathrm{PI})=2.5$, and $T_{\mathrm{g}}=207^{\circ} \mathrm{C}$.

Blend samples of ternary polymers were prepared by using solution blending and film-casting. For convenience, the three polymers, PS, iPS, and PCHMA (or PS, iPS, PPO), are labeled in order of Roman numerals as Component-I, -II, and -III, respectively, in the ternary blend, and the weight ratios in ternary blends are indicated in the exact order of I/II/III. The ternary blend samples in this study were prepared by mixing in solvents, to be followed with film-casting at $45^{\circ} \mathrm{C}$. Tetrahydrofuran (THF) or toluene was used as a solution-blending medium in preparation of all PS/iPS/PCHMA and PS/iPS/PPO ternary blend samples, respectively. The polymers were first weighed respectively and dissolved into THF (or toluene) with continuous stirring using a magnet. Subsequently, the resulting polymer solution was poured into a flat aluminum or glass mold kept at $45^{\circ} \mathrm{C}$. The solvent in the cast samples was first vaporized under a hood at controlled temperature, followed by residual solvent removal in a vacuum oven for $48 \mathrm{~h}(2 \mathrm{~d})$ at $80^{\circ} \mathrm{C}$. Subsequent vacuum degassing at an even higher temperature of $110^{\circ} \mathrm{C}$ was performed on the castfilm samples for two more days to ensure removal of residual solvent.

\section{Apparatus}

Differential Scanning Calorimetry (DSC). The glass transition temperatures, crystallization, and melting of the ternary blend samples were measured with a differential scanning calorimeter (PerkinElmer DSC-7) equipped with an intracooler and a computer for data acquisition/analysis. All $T_{\mathrm{g}}$ and $T_{\mathrm{m}}$ measurements were carried out at a scan rate of $20^{\circ} \mathrm{C} / \mathrm{min}$ and $10^{\circ} \mathrm{C} / \mathrm{min}$, respectively. The $T_{\mathrm{g}}$ values were taken as the onset of the transition (the change of the specific heat) in the DSC thermograms. For a uniform thermal history, all DSC thermograms shown in the graphs are the results of second runs after quenching from above the prospective $T_{\mathrm{g}}$. For isothermal crystallization, each sample was heated up to $240{ }^{\circ} \mathrm{C}$ for $5 \mathrm{~min}$ in order to eliminate crystalline residues formed during the preparation procedure. The samples were then quenched at a rate of $320^{\circ} \mathrm{C} / \mathrm{min}$ to a desired crystallization temperature, $T_{\mathrm{c}}$, and held there for $8 \mathrm{~h}$.

Polarized-light optical microscopy (POM). A polarized-light optical microscope (Nikon Optiphot-2 POL) was used for observation of optical phase behavior. The as-cast blends were spread as thin films on glass slides, dried properly in a temperature-controlled oven before they were examined using the optical microscope. Temperature-induced phase transition in the blends was performed by placing the sam- 
ples on a microscope heating stage (Linkam THMS600 with TP-92 temperature programmer), with a programmed heating rate (or cooling) of approximately $2{ }^{\circ} \mathrm{C} / \mathrm{min}$ from room temperature up to $300^{\circ} \mathrm{C}$.

Scanning electron microscopy (SEM). Blend samples were also examined using a scanning electron microscope (SEM, JEOL JXA840) for revealing possible sub-micro phase domains. The cast samples, prepared at $45^{\circ} \mathrm{C}$, were examined using SEM after preliminary optical microscopy characterization. The blend film samples for scanning electron microscopy were thick enough so that fracture surface of the thickness (cross section) could be conveniently examined. The fractured blend samples were coated with gold by vapor deposition using a sputter.

\section{RESULTS AND DISCUSSION}

\section{Glass Transitions Behavior}

Figure 1a shows the DSC thermograms $\left(20^{\circ} \mathrm{C} /\right.$
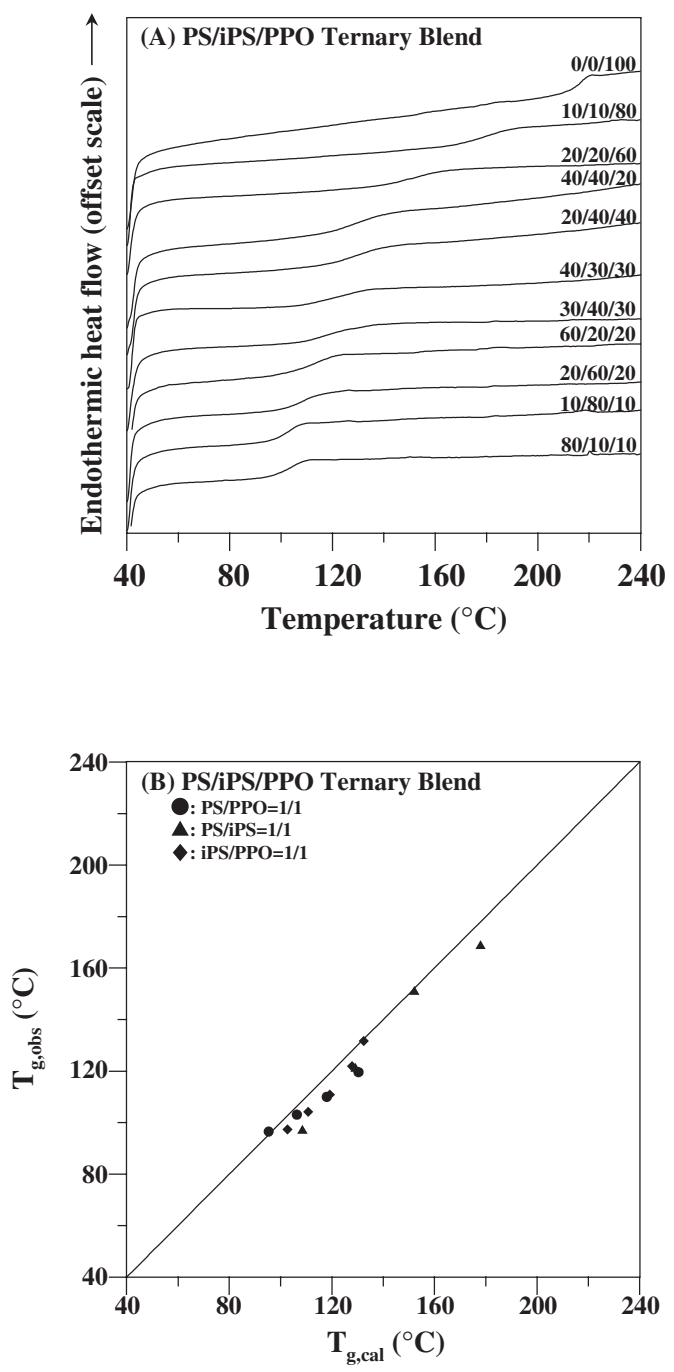

Figure 1. (A) DSC thermograms showing single $T_{\mathrm{g}}$ in each of the PS/iPS/PPO blend of various compositions, and (B) experimental $T_{\mathrm{g}}$ data by DSC $v s$. the Fox equation. min) revealing only a single $T_{\mathrm{g}}$ for each of the ternary PS/iPS/PPO blend samples. DSC runs were performed on the ternary blend samples to cover as wide a ternary composition range as possible; but for brevity, only DSC traces of several representative blend compositions are displayed in this figure. The $T_{\mathrm{g}}$ 's of these three polymers differ significantly and are spaced in a wide temperature range $\left(90^{\circ} \mathrm{C}, 95^{\circ} \mathrm{C}\right.$ and $207^{\circ} \mathrm{C}$, respectively) that made it straightforward in applying the $T_{\mathrm{g}}$ criteria for assessing the phase behavior. For all samples investigated in this study, a distinct single $T_{\mathrm{g}}$ was seen, and it apparently varied with compositions. Thus, the DSC thermal evidence clearly indicates this is a miscible ternary blend system. Figure $1 \mathrm{~b}$ shows comparison of the experimental $T_{\mathrm{g}}$ data for the ternary PS/iPS/PPO blends with the $T_{\mathrm{g}}$ values calculated from the Fox equation. The agreement between the experimental data with the Fox model prediction is reasonably good within the entire ternary composition ranges investigated. In addition, single $T_{\mathrm{g}}$ was also noted in the ternary PS/iPS/ PCHMA blends, which has been discussed and reported earlier.

\section{Phase Morphology}

The cast films of all PS/iPS/PCHMA compositions were examined using POM, which revealed apparently clear and homogeneous structure at above the melting temperature of iPS. Blends with PS-rich or PCHMA-rich compositions contained virtually no iPS crystals, and these blend samples at ambient temperature were clear and free of any heterogeneous domains when examined using the optical microscope at the maximum magnification (not shown here for brevity). In addition to the optical microscopy characterization, blend morphology was also observed using SEM. Figure 2 shows the SEM micrographs of six representative PS/iPS/PCHMA blend samples: (A) 80/10/10, (B) 40/30/30, (C) 10/80/10, (D) 30/40/ 30, (E) $10 / 10 / 80$, and (F) $35 / 35 / 30$ (wt \%), respectively. Therefore, SEM characterization of phase morphology was demonstrated on the fully amorphous PS/iPS/PCHMA blend compositions. The six graphs clearly show no discernible heterogeneity in these samples, which is additional supportive evidence on phase homogeneity in PS/iPS/PCHMA blend system. Furthermore, Figure 3 shows the SEM graphs to illustrate the homogeneity in the miscible PS/iPS/PPO blend. The SEM characterization revealed no discernible heterogeneity in the solution-cast PS/iPS/PPO blend samples in a wide composition range. Although the SEM characterization revealed absence of sub-micron fine domains, it could not be taken as direct indication of phase miscibility. However, at the resolution of SEM no discernible micro-heterogeneity existed in 

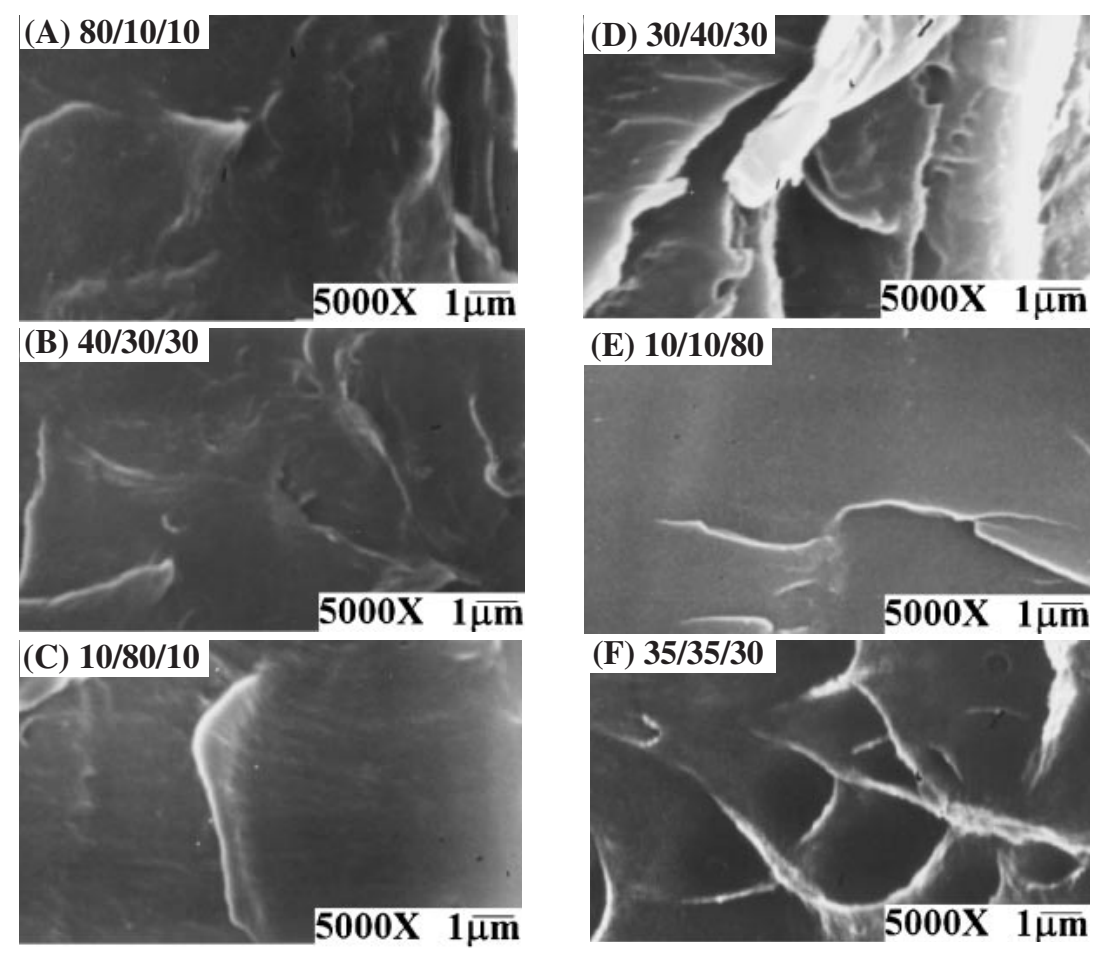

Figure 2. SEM micrographs on fractured surface of the PS/iPS/PCHMA blends: (A) 80/10/10; (B) 40/30/30; (C) 10/80/10; (D) 30/ 40/30; (E) 10/10/80; and (F) 35/35/30.

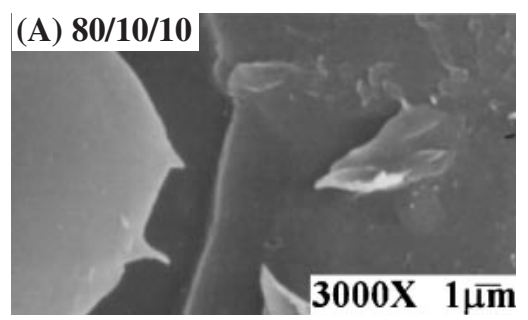

(B) 20/40/40

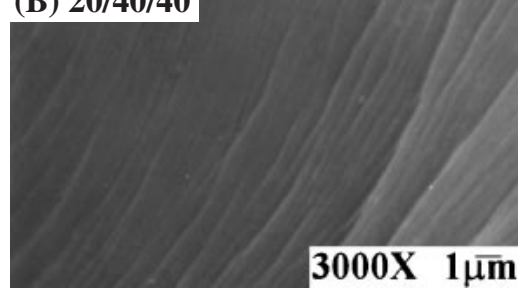

(C) $20 / 60 / 20$

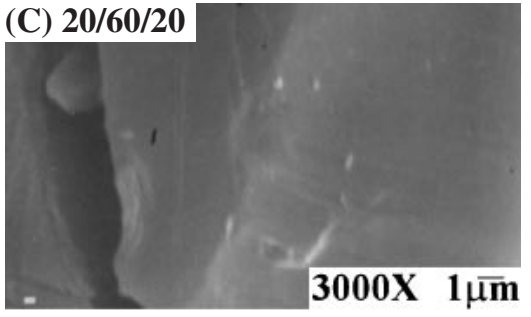

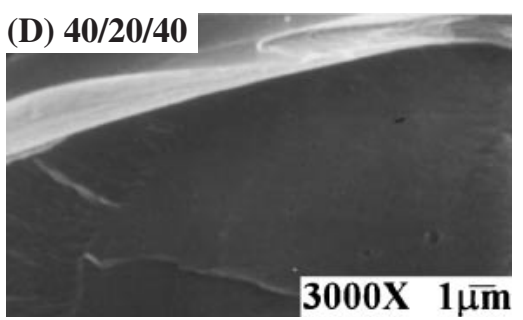
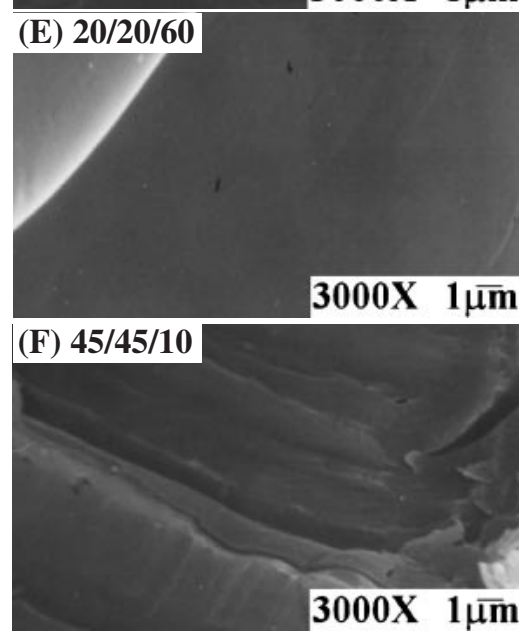

Figure 3. SEM micrographs on fracture surface of the PS/iPS/PPO blend samples: (A) 80/10/10; (B) 20/40/40; (C) 20/60/20; (D) 40/20/40; (E) 20/20/60; and (F) 45/45/10.

the PS/iPS/PPO blend, whose miscibility was already indicated by the glass transition criteria.

\section{Cloud-point Transition in PS/iPS/PChMA Blend}

Possible effects of temperature on phase transition (from homogeneous to phase separation upon heating) in the miscible ternary blends were examined using the optical microscope with a heating stage. The originally miscible PS/iPS/PCHMA blends were heated slowly $\left(\mathrm{ca} .2^{\circ} \mathrm{C} / \mathrm{min}\right)$ to higher temperatures for ob- 
(A) 40/40/20
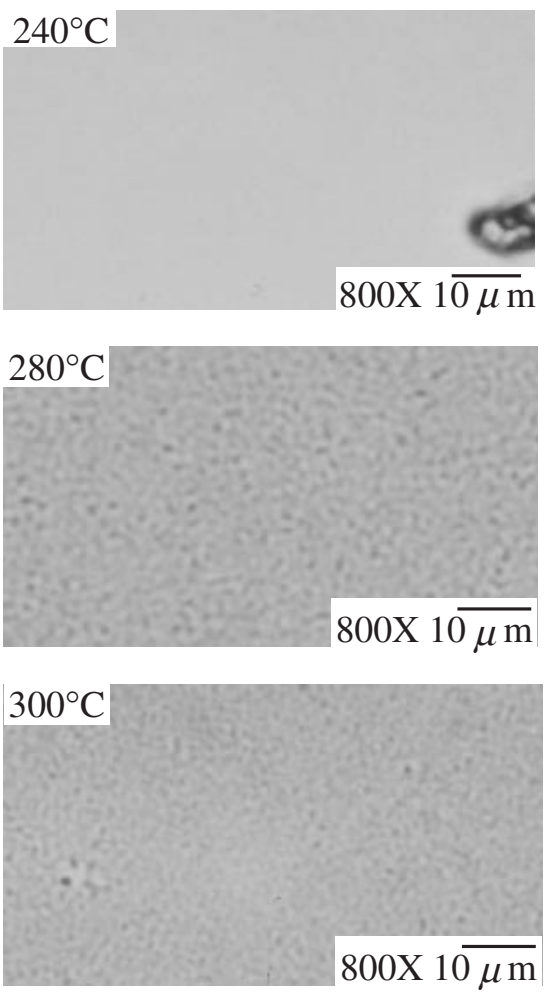

(B) $35 / 35 / 30$
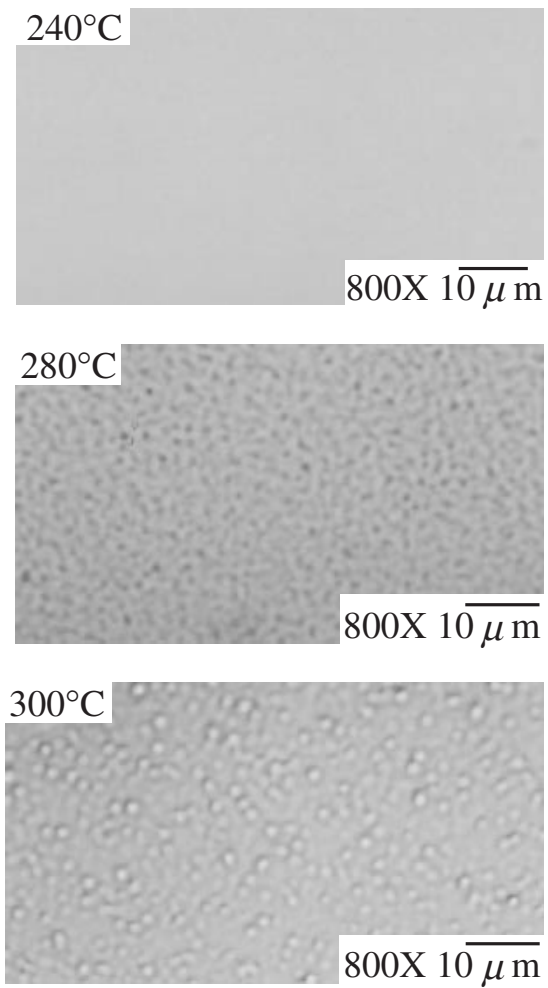

Figure 4. The optical micrographs $(800 \times)$ reveal the phase transitions in the PS/iPS/PCHMA ternary blends of compositions: (A) 40/ $40 / 20$, and (B) $35 / 35 / 30$, at various temperatures $\left(240,280\right.$, and $\left.300^{\circ} \mathrm{C}\right)$.

serving phase change. Figure 4 shows the actual optical micrographs $(800 \times)$ revealing the phase domains and phase separation transitions in the PS/iPS/ PCHMA ternary blends at increasing temperatures (240, 280, and $300^{\circ} \mathrm{C}$ ) for two different compositions: (A) $40 / 40 / 20$, and (B) $35 / 35 / 30$. Both blends exhibited phase domains with similar spinodal decomposition upon heating to $300{ }^{\circ} \mathrm{C}$. Upon increase of temperature from 240 to $300^{\circ} \mathrm{C}$, a transition is apparent from original homogeneity (transparent and no phase separation) to initial phase separation of a worm-like, interconnected fibrillar pattern to the final morphology owing to the percolation-to-cluster transition pattern into discrete spherical domains. The pattern of phase domains suggests similar spinodal decomposition upon heating to above the cloud point for both compositions. Samples of other compositions were examined, which are not shown for brevity.

The result of cloud-point temperatures and miscibility/immiscibility boundary for the ternary PS/ iPS/PCHMA blend is summarized in Figure 5. Cloud point transition (from homogeneity to phase separation, etc.) was found in most of the blend compositions, but the temperature of cloud transition (phase separation) varied from a low temperature of $206^{\circ} \mathrm{C}$ to a quite high temperature of $296^{\circ} \mathrm{C}$ depending on the ternary blend compositions.
For three-polymer blend systems, it may be interesting to examine the variation trend of cloud-points as the content of one of three components was varied. Figure 6 shows the cloud point curves for the ternary PS/iPS/PCHMA blend. In Graph-I for Tieline-I of ternary compositions, the top triangle diagram shows the ternary blend compositions whose cloud points are to be plotted. The ternary blend compositions were chosen in a way that the relative PCHMA/iPS contents were kept constant at equal proportions (1:1) but the content of PS was varied from low to high $(10-90 \mathrm{wt} \%)$. A concave cure was resulted, suggesting the effect of increasing of the components (PCHMA in this case) first led to a lower cloud point, but then the trend is reversed. Most of the PS/iPS/ PCHMA blend compositions were observed to undergo phase separation at elevated temperature. It can also be seen from the figure that both PS/PCHMA and iPS/PCHMA binary blends exhibit LCST behavior. The existence of a minimum on the cloud point surface means that there is a large decrease in the ternary LCST to those of the binary blends, indicating the reduced miscibility for ternary compositions. However, it is interesting to note that there is no LCST near the right side of triangle. 


\section{iPS (Component II)}

PCHMA

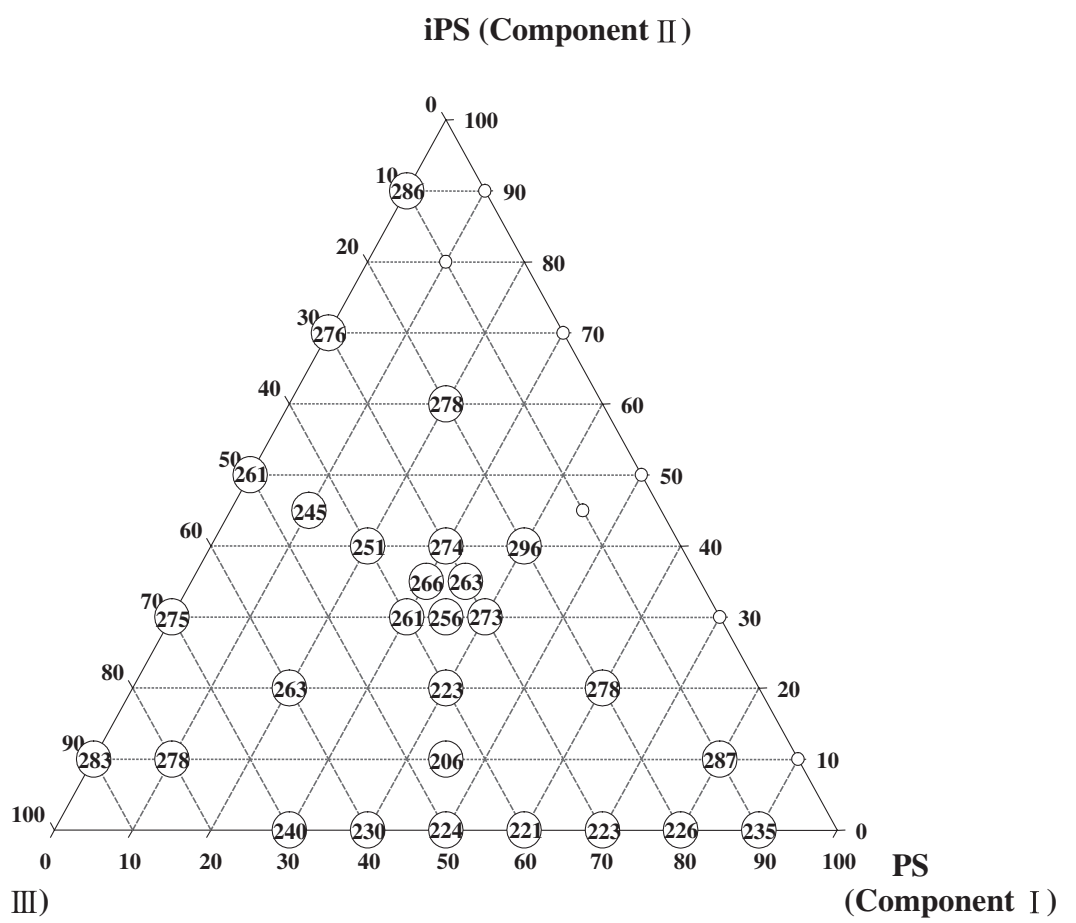

Figure 5. Summary of cloud points for PS/iPS/PCHMA ternary blend.

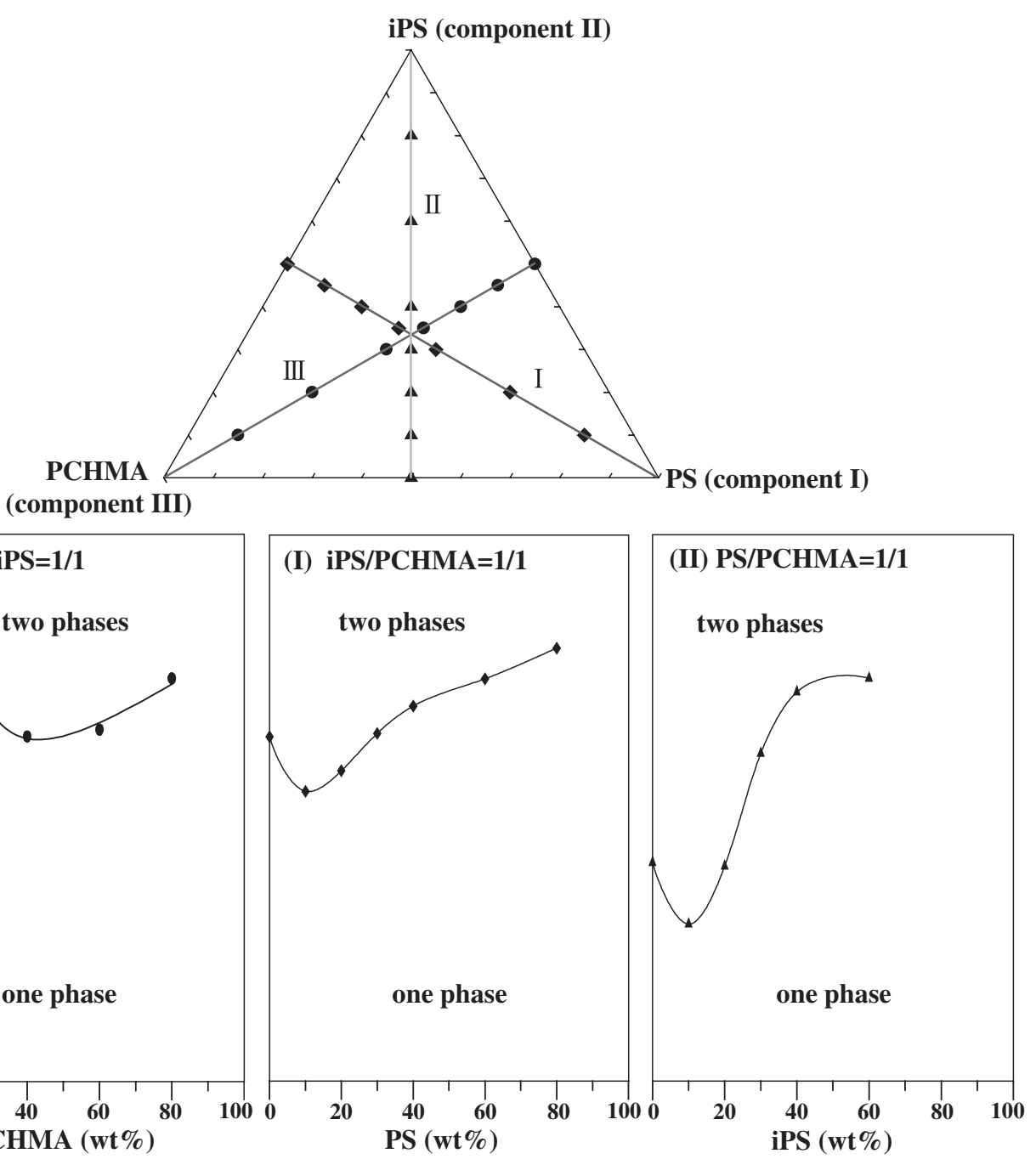

Figure 6. Representation of cloud point curves for PS/iPS/PCHMA ternary blend: two-dimensional view. 


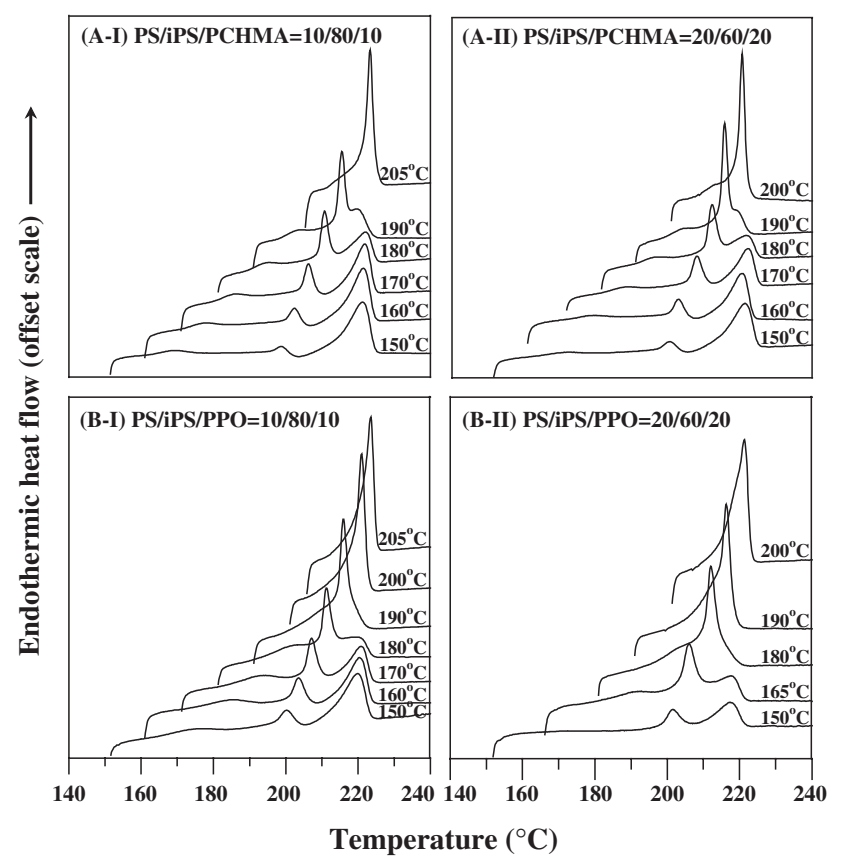

Figure 7. $\mathrm{DSC}$ traces $\left(10^{\circ} \mathrm{C} / \mathrm{min}\right)$ of (A) PS/iPS/PCHMA, and (B) PS/iPS/PPO ternary blends melt-crystallized at various temperatures for $8 \mathrm{~h}$.

\section{Melting Point Depression of iPS in Ternary Blends}

The analysis of the melting behavior of a crystalline component in semi-crystalline polymer blends is an important tool in assessing polymer miscibility. In miscible blends, the melting point of the crystalline component is usually lowered with respect to the pure polymer as a result of thermodynamically favorable interactions. Figure 7 shows the DSC traces $\left(10^{\circ} \mathrm{C} /\right.$ min) of (A) PS/iPS/PCHMA, and (B) PS/iPS/PPO ternary blends. The melt crystallization temperatures were chosen to range from $150^{\circ} \mathrm{C}$ to $205^{\circ} \mathrm{C}$, and insurance was made that there was no transient crystallization occurring during cooling from the melt to crystallization temperature $\left(T_{\mathrm{c}}\right)$ in these ternary blend systems. It shows three melting peaks with lowest melting peak $T_{\mathrm{m}, \mathrm{I}}$, the intermediate melting peak $T_{\mathrm{m}, \mathrm{II}}$, and the highest melting peak $T_{\mathrm{m}, \mathrm{III}}$ for $T_{\mathrm{c}}$ from $150^{\circ} \mathrm{C}$ to $205^{\circ} \mathrm{C}$. The lowest temperature peak, the so-called annealing peak, shifts to higher temperature with increasing $T_{\mathrm{c}}$ and appears at $c a .10-15^{\circ} \mathrm{C}$ above $T_{\mathrm{c}}$. Its magnitude also increases slightly with the increase of $T_{\mathrm{c}}$. The highest temperature peak corresponds to the melting of crystallites that are formed and re-crystallized during heating. The intermediate peak corresponds to the melting of crystallites formed at $T_{\mathrm{c}}$ by a primary crystallization process. ${ }^{30,31} T_{\mathrm{m}, \mathrm{II}}$ increases in intensity as $T_{\mathrm{c}}$ increases, while the highest temperature peak decreases in intensity. For the case of $T_{\mathrm{c}}>170^{\circ} \mathrm{C}$, the re-crystallization process is somewhat inhibited, therefore, the re-crystallization exotherm cannot be distinctly seen, as shown in

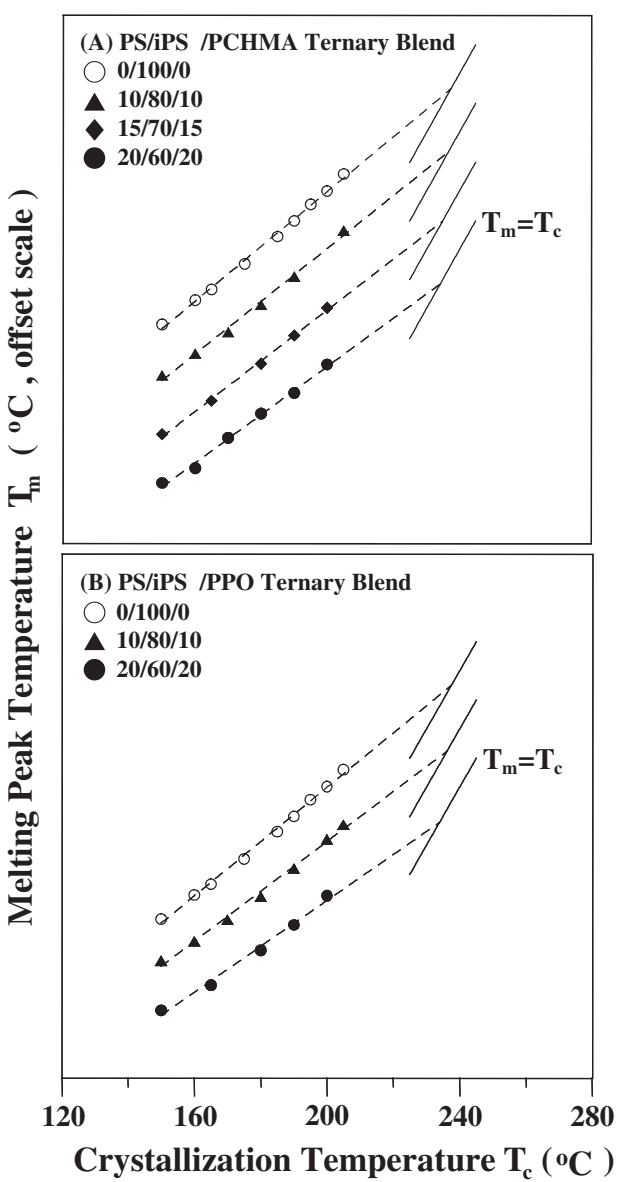

Figure 8. Hoffman-Weeks plot for (A) PS/iPS/PCHMA blends of PS/PCHMA weight ratio of $1 / 1$, and (B) PS/iPS/ PPO blend of PS/PPO weight ratio of $1 / 1$.

Figure 7 . When $T_{\mathrm{c}}$ increases above $200^{\circ} \mathrm{C}$, only one single melting peak can be observed, which shifts to higher temperature as $T_{\mathrm{c}}$ increases.

Furthermore, the equilibrium melting points were determined from the Hoffman-Weeks plots. Figure 8 shows Hoffman-Weeks plots with extrapolation of the melting temperature (in offset $y$-axis) for the PS/iPS/PCHMA and PS/iPS/PPO blend samples, each piece of experimental data is obtained by isothermal crystallization for $8 \mathrm{~h}$. As observed, a linear correlation between $T_{\mathrm{m}}$ and $T_{\mathrm{c}}$ is obtained. The extrapolated Hoffman-Weeks plots yield the equilibrium melting points of crystals of infinite size where surface effects are negligible. ${ }^{18}$ The equilibrium melting temperature of the blends decreases as the PCHMA (or PPO) composition increases from 237.5 to $234.2^{\circ} \mathrm{C}$ (or 237.5 to $233.9^{\circ} \mathrm{C}$ ). This is report of the observation of melting point depression in these two ternary blend systems.

As in the case of the binary polymer mixtures, the melting point depression of a crystallizable component in a ternary solution can be readily derived from the relevant equations. ${ }^{32}$ The key arguments in the derivation begin with the assumption by Hildebrand 


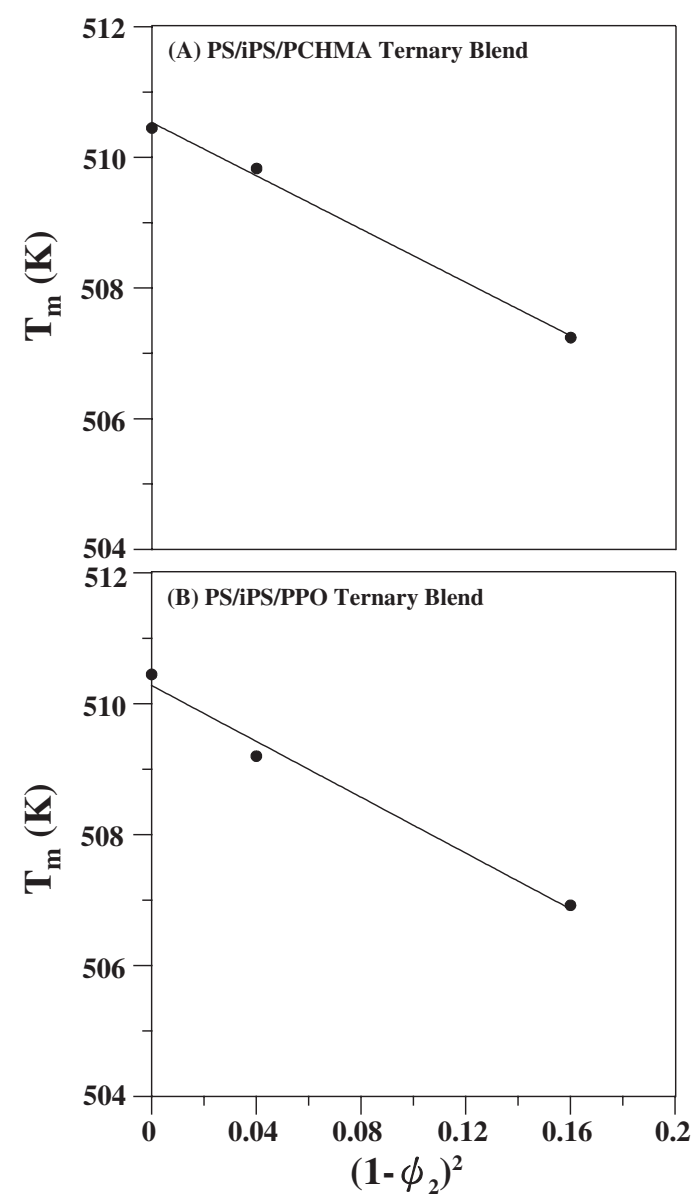

Figure 9. $T_{\mathrm{m}}$ of iPS in ternary blends: (A) PS/PCHMA of weight ratio of $1 / 1$, and (B) PS/PPO weight ratio of $1 / 1$.

and $\operatorname{Scott}^{33}$ that the heat of mixing, $\Delta H_{\text {mix }}$, of a multi-component system can be described in terms of binary interaction coefficients by

$$
\Delta H_{\text {mix }}=V\left\{\sum_{i} \sum_{j \neq i} B_{i j} \phi_{i} \phi_{j}\right\}
$$

where $V$ is the system volume, $B_{i j}$ is the interaction coefficient which determines the sign and magnitude of the heat of mixing of $i$ with $j$, and $\phi_{i}$ is the volume fraction of component $i$ in the mixture. As in the case of binary mixtures, $\Delta H_{\text {mix }}=0$ becomes the criterion for predicting a boundary between single-phase and multiphase behavior. ${ }^{34,35}$

For a ternary mixture of components 1,2 , and 3 , the partial molar enthalpy of component $2, \Delta \bar{H}_{2}$, becomes $^{36,37}$

$$
\begin{aligned}
\Delta \bar{H}_{2} & =\partial \Delta H_{\text {mix }} / \partial n_{2} \\
& =\tilde{V}_{2}\left\{B_{12} \phi_{1}^{2}+B_{23} \phi_{3}^{2}+\Delta B \phi_{1} \phi_{3}\right\}
\end{aligned}
$$

where

$$
\Delta B=B_{12}+B_{23}-B_{13}
$$

and $\tilde{V}_{2}$ is the molar volume of component 2. Using eq 2 to equate the difference in chemical potentials between a crystalline polymer unit and the same unit in the pure liquid state, ${ }^{38}$ we obtain:

$$
T_{\mathrm{m}}=T_{\mathrm{m}}^{0}\left\{1+\left(\Delta \tilde{H}_{2} / \tilde{V}_{2}\right)\left(v_{2 u} / \Delta h_{2 u}\right)\right\}
$$

where $v_{2 u}$ and $\Delta h_{2 u}$ are volume and heat of fusion, respectively, per unit of crystalline component 2. $T_{\mathrm{m}}$ and $T_{\mathrm{m}}^{0}$ are the melting temperature of component 2 in the blend and in the pure state, respectively. Combination of eqs 2-4 yields a well-known result: ${ }^{38,39}$

$$
T_{\mathrm{m}}=T_{\mathrm{m}}^{0}\left\{1+B\left(v_{2 u} / \Delta h_{2 u}\right)\right\}\left(1-\Psi_{2}\right)^{2}
$$

where

$$
B=B_{12} \Psi_{1}^{2}+B_{23} \Psi_{2}^{2}+\Delta B \Psi_{1} \Psi_{3}
$$

and

$$
\Psi_{i}=\phi_{i} /\left(1-\phi_{2}\right)
$$

For a miscible binary blend of crystalline polymer 2 and amorphous polymer $1, \phi_{3}=0, \Psi_{3}=0$, $\phi_{1}=\left(1-\phi_{2}\right), \Psi_{1}=1$, and $B$ in eq 5 is just $B_{12}$, the interaction parameter describing the heat of mixing between 2 and 1. The generally observed decline in the melting temperature with increasing contents of the amorphous diluent leads to conclusion that $B_{12}$ and $\Delta H_{\text {mix }}$ are negative for miscible binary blends. ${ }^{40-42}$ Equation 5 suggests that the parameter, $B$, can be evaluated in exactly the same way for a ternary mixture as for a binary blend by determining the slope of the $T_{\mathrm{m}}$ versus the amorphous volume fraction construction. Evaluation of $B$ at PS/PCHMA (or PS/PPO) ratio of 1 , should then allow evaluation of unknown interaction parameter for PS/PCHMA (or PS/PPO), $B_{13}$ (or $B_{13}^{\prime}$ ), via eqs 3 and 6 .

Figure 9 shows the melting point depression of iPS in the PS/iPS/PCHMA and PS/iPS/PPO ternary blends. According to eq 5, the B values for PS/iPS/ PCHMA and PS/iPS/PPO were determined from the slope of the straight line, respectively. The values of $92.857 \mathrm{~cm}^{3} / \mathrm{mol}$ and $8 \mathrm{~kJ} / \mathrm{mol}$ were used for $v_{2 u}$ and $\Delta h_{2 u}$, respectively, in eq 5 . From the slopes, two overall interaction parameters in $\mathrm{PS} / \mathrm{iPS} /$ PCHMA and PS/iPS/PPO ternary blends were determined: $B=-3.44 \mathrm{~J} / \mathrm{cm}^{3}$ for a PS/PCHMA ratio of 1 and $B=-3.60 \mathrm{~J} / \mathrm{cm}^{3}$ for a PS/PPO ratio of 1 , indicating that both PS/iPS/PCHMA and PS/iPS/PPO are miscible.

To apply eqs 3 and 6 to the present ternary blends, one denotes PS with subscript-1, iPS with 2 , and PCHMA (or PPO) with 3. There are a total of five binary-pair interaction energy parameters for the two PS/iPS/PCHMA and PS/iPS/PPO ternary blends. Among them, $B_{12}$ (PS/iPS), and $B_{23}^{\prime}$ (iPS/PPO) have been determined in the literature ${ }^{21,22}$ by combining the equilibrium melting point depression and a binary interaction model. The values were $B_{12}=-1.095 \mathrm{~J} /$ 


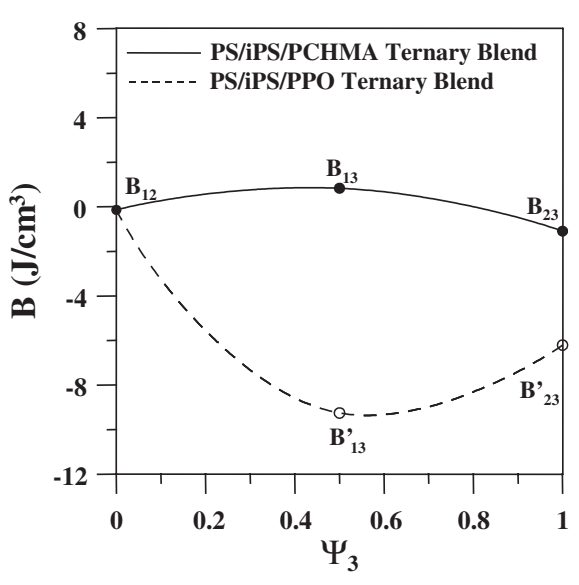

Figure 10. Interaction parameter $B v s$. the fraction of crystallizing iPS in the amorphous diluents comprising (-) aPS and PCHMA; and (---) aPS and PPO.

$\mathrm{cm}^{3}$, and $B_{23}^{\prime}=-6.213 \mathrm{~J} / \mathrm{cm}^{3}$. The value of $B_{23}$ corresponding to the interaction between iPS and PCHMA was directly obtained from the melting point depression of iPS in the iPS/PCHMA binary blend. The value of $B_{23}$ is $-1.095 \mathrm{~J} / \mathrm{cm}^{3}$, as evaluated in an earlier study. ${ }^{23}$ However, two parameters $B_{13}$ and $B_{13}^{\prime}$ still remained unknown. In order to evaluate the two unknown parameters, one can use eqs 3 and 6 . The overall interaction parameter $B$ has been evaluated from the equilibrium melting point depression at a given $\Psi_{3}$, which corresponding to the PS/PCHMA or PS/PPO ratios of 1 . Upon substitution of two overall interaction parameters and the corresponding values into eqs 3 and 6 , the unknown parameters $B_{13}$ and $B_{13}^{\prime}$ can be evaluated by solving two simultaneous equations since the values of three parameters $B_{12}$, $B_{23}$, and $B_{23}^{\prime}$ have been known. The estimated values were $B_{13}=0.826 \mathrm{~J} / \mathrm{cm}^{3}$ and $B_{13}^{\prime}=-9.259 \mathrm{~J} / \mathrm{cm}^{3}$. Figure 10 shows interaction parameter $B v s$. the fraction of iPS in the amorphous dilute. It is interesting to note that the interaction parameter of PS/PCHMA binary blend is small positive. The positive value of $B_{13}$ suggests that PS/PCHMA is immiscible at $240^{\circ} \mathrm{C}$, which is above LCST. In addition, the value of $B_{13}^{\prime}$ is a large negative, indicating that PS/PPO remains miscible at temperature up to $240^{\circ} \mathrm{C}$.

\section{Binary Interactions and Ternary Miscibility}

To extent that the primary requirement for binary blend miscibility is a negative heat of mixing between blend components, it seems reasonable that the same criterion should hold in multi-component polymer blends. Thus, one should be able to estimate the locus of blend compositions that define the boundary between miscible and immiscible behavior by setting eq 1 to zero. The results of the ternary PS/iPS/ PCHMA and PS/iPS/PPO systems allow an experi- mental check on the assumption that $\Delta H_{\text {mix }} \leq 0$ is a sufficient criterion for miscibility within the limit of uncertainty associated with the $B_{i j}$ parameters determined above.

The locus of ternary compositions is easily determined by combining eq 1 , with $\Delta H_{\text {mix }}=0$, and the equation of incompressibility, $\sum \phi_{i}=1$, to yield: ${ }^{36,37}$

$$
\phi_{2}=R B_{13} /\left[R B_{13}+(R+1) /\left(R B_{12}+B_{23}\right)\right]
$$

where

$$
\begin{aligned}
\phi_{3} & =\left(1-\phi_{2}\right) /(1+R) \\
R & =\phi_{1} / \phi_{3}
\end{aligned}
$$

The calculation requires specifying a value of $R$, from which the volume fraction of iPS, $\phi_{2}$, is calculated by eq 8 , that of PCHMA (or PPO), $\phi_{3}$, is calculated by eq 9 , and that of PS, $\phi_{1}$, is calculated by eq 10 . Figure 11 shows the calculated miscibility boundary with the estimates of miscible behavior obtained experimentally at $240^{\circ} \mathrm{C}$ in PS/iPS/PCHMA and PS/ iPS/PPO ternary blend systems. The calculated phase boundary is a bit higher in temperatures than the ex-
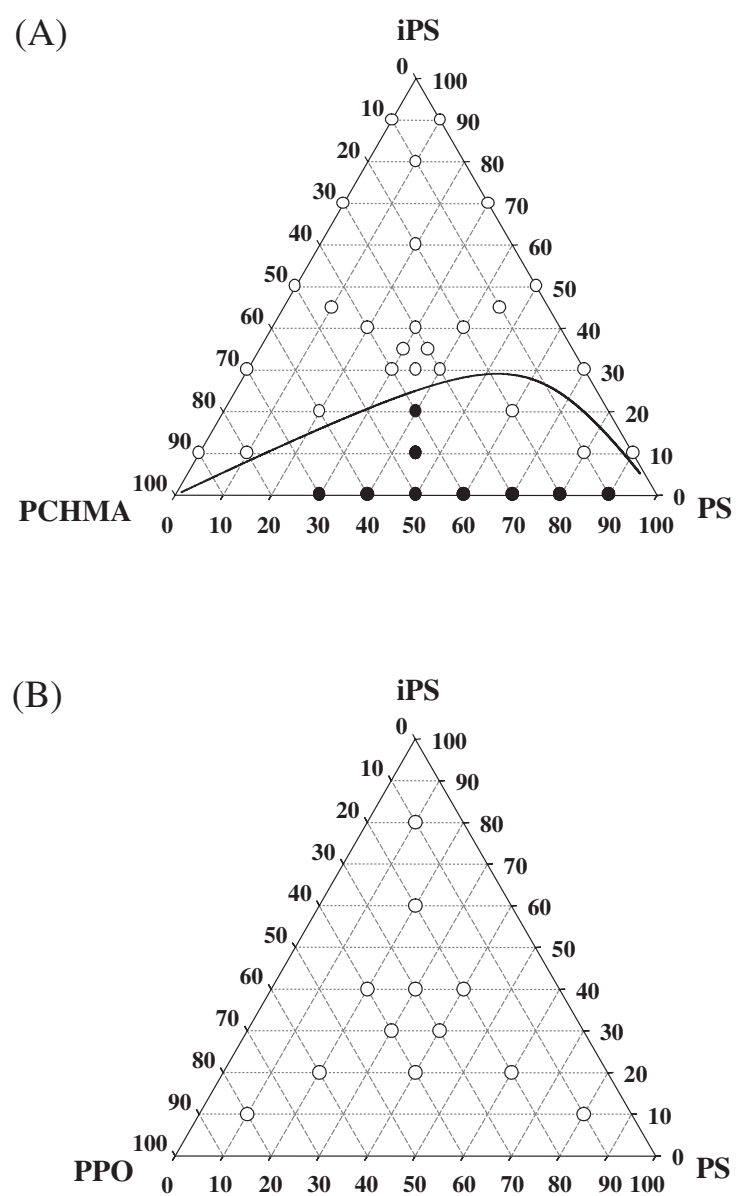

Figure 11. Ternary phase diagrams at $240^{\circ} \mathrm{C}$ for: (A) PS/ iPS/PCHMA, and (B) PS/iPS/PPO ternary blends. Open circles represent transparent/miscible blends while filled circles represent cloudy/separated blends. The solid curve denotes the theoretically calculated miscibility-immiscibility boundary. 
perimental results for the PS/iPS/PCHMA ternary blend. In other words, the calculated immiscibility region is slightly larger than the experimental results. Besides the significant enthalpy contribution, the marginal entropic gain on mixing also contributes to the decreasing free energy. As a result, the experimental miscibility region is enlarged with respect to the calculated result. The phase diagram of PS/iPS/PCHMA is asymmetric, indicating a large $\Delta \chi$ effect in this ternary blend system. In contrast to the ternary PS/iPS/ PCHMA blend system, an immiscibility-loop is not present in the PS/iPS/PPO blend system at $240^{\circ} \mathrm{C}$.

\section{CONCLUSIONS}

The miscibility, morphology and phase behavior of two ternary blend systems, PS/iPS/PCHMA and PS/ iPS/PPO, were investigated and compared. Ternary blend miscibility with complete phase homogeneity in the entire composition range was demonstrated in this study using the criteria of thermal behavior and microscopic characterization. Both ternary blend systems of PS/iPS/PCHMA and PS/iPS/PPO were found to be fully miscible, with no closed-loop immiscibility. LCST behavior was not seen in the ternary PS/iPS/PPO blend system. However, the PS/iPS/ PCHMA ternary miscible blend exhibited a lower critical solution temperature (LCST) equivalent to that observed for the binary PS/PCHMA or iPS/PCHMA miscible blend systems, and the LCST for the PS/ iPS/PCHMA ternary miscible blend system was significantly lower than those for any of the three binary miscible pairs. This suggests that the interactions in the ternary system may have been disrupted to a lower level in comparison with those among the binary miscible pairs (PS/iPS, iPS/PCHMA, or PS/PCHMA).

In addition, the ternary phase behavior was further compared with the five possible pairs of binary blends that can be formed from these four different polymer constituents (PS, iPS, PCHMA, and PPO), where all the five binary blends have been known to be miscible as already documented in the literature. Melting point depression analysis of iPS in the ternary blends yielded the estimate of $B=-3.44$ and $B=-3.6 \mathrm{~J} / \mathrm{cm}^{3}$ for the PS/iPS/PCHMA and PS/iPS/PPO ternary blend systems, respectively. Knowledge of the interaction parameters for the miscible pairs allowed us to obtain the interaction energy $B_{13}=0.826 \mathrm{~J} / \mathrm{cm}^{3}$ between PS and PCHMA or $B_{13}^{\prime}=-0.9259 \mathrm{~J} / \mathrm{cm}^{3}$ between PS and PPO. Good correlation was found between the fitted $B_{13}$ and $B_{13}^{\prime}$ from the thermodynamic theory and the experimental data for the PS/PCHMA and PS/ PPO binary blend pairs. The positive value of $B_{13}$ suggests that PS/PCHMA blend system is immiscible at $240^{\circ} \mathrm{C}$, which is a temperature above LCST. The ther- modynamic theory works better for binary miscible pairs, but it can be extended to ternary polymer blends to provide a satisfactory explanation of the experimental trend. Phase diagrams for both ternary systems at elevated temperatures were constructed and compared with the experimental data. The experimental miscibility region is slightly larger than but still quite comparable with respect to the calculated result. The calculated cloud-point transition region in the phase diagram of PS/iPS/PCHMA is of asymmetry, indicating a more pronounced $\Delta \chi$ effect in this ternary blend system in comparison to the PS/iPS/PPO system.

\section{REFERENCES}

1. G. R. Brannock and D. R. Paul, Macromolecules, 23, 5240 (1990).

2. S. N. Yau and E. M. Woo, Macromol. Rapid Commun., 17, 615 (1996).

3. Y. C. Tseng and E. M. Woo, Macromol. Rapid Commun., 19, 215 (1998).

4. J. M. G. Cowie, G. Li, and I. J. McEwen, Polymer, 25, 5518 (1994).

5. J. A. Pomposo, E. Calahorra, I. Eguiazabal, and M. Cortazar, Macromolecules, 26, 2104 (1993).

6. W. H. Jo, Y. K. Kwon, and I. H. Kwon, Macromolecules, 24, 4708 (1991).

7. K. E. Min, J. S. Chiou, J. W. Barlow, and D. R. Paul, Polymer, 28, 1721 (1987).

8. S. H. Goh and X. Ni, Polymer, 40, 5733 (1999).

9. H. Zhang, D. E. Bhagwagar, J. F. Graf, P. C. Painter, and M. M. Coleman, Polymer, 25, 5379 (1994).

10. B. K. Hong, J. Y. Kim, W. H. Jo, and S. C. Lee, Polymer, 38, 4373 (1997).

11. S. W. Kuo, C. C. Lin, and F. C. Chang, Macromolecules, 35, 278 (2002).

12. T. K. Kwei, H. L. Frisch, W. Radigan, and S. Vogel, Macromolecules, 10, 157 (1977).

13. T. Nishi, T. Suzuki, N. Matsuzawa, S. Michizono, and H. Tanaka, Polym. Prepr. (Am. Chem. Soc., Div. Polym. Chem.), 28, 110 (1987).

14. K. E. Min, J. S. Chiou, J. W. Barlow, and D. R. Paul, Polymer, 29, 1052 (1987).

15. R. E. Prud'homme, Polym. Eng. Sci., 22, 90 (1982).

16. C. Lau, Z. Zhong, and Y. Mi, Macromolecules, 31, 7291 (1998).

17. J. D. Honeycutt, Macromolecules, 27, 5377 (1994).

18. J. Stoelting, F. E. Karasz, and W. MacKnight, Polym. Eng. Sci., 10, 133 (1970).

19. A. R. Shultz and B. M. Beach, Macromolecules, 7, 902 (1974).

20. W. M. Prest, Jr. and R. S. Porter, J Polym. Sci., Part A-2: Polym. Phys., 10, 1639 (1972).

21. J. Plans, W. J. MacKnight, and F. E. Karasz, Macromolecules, 17, 810 (1984).

22. J. P. Runt, Macromolecules, 14, 420 (1981).

23. F. H. Jang and E. M. Woo, Polymer, 40, 2231 (1999).

24. C. Friedrich, C. Schwarzwalder, and R.-E. Riemann, 
Polymer, 37, 2499 (1996).

25. L. L. Chang and E. M. Woo, J. Polym. Sci., Polym. Phys. Ed., 41, 772 (2003).

26. L. L. Chang and E. M. Woo, Polymer, 45, 6909 (2004).

27. T. K. Kwei, G. D. Patterson, and T. T. Wang, Macromolecules, 9, 780 (1976).

28. O. Olabisi, Macromolecules, 8, 316 (1975).

29. J. M. Jonza and R. S. Porter, Macromolecules, 19, 1946 (1986).

30. H. Berghmans and N. Overbergh, J. Polym. Sci., Polym. Phys. Ed., 48, 4068 (1977).

31. P. J. Lemstra, T. Kooistra, and G. Challa, J. Polym. Sci., Part A-2: Polym. Phys., 10, 823 (1972).

32. R. L. Scott, J. Chem. Phys., 17, 279 (1949).

33. J. H. Hildebrand and R. L. Scott, "The Solubility of Nonelectrolytes," Dover, New York, N.Y., 1964.

34. J. W. Barlow and D. R. Paul, Ann. Rev. Mater. Sci., 11, 299
(1981).

35. D. R. Paul and J. W. Barlow, Polymer, 25, 487 (1984).

36. W. H. Christiansen, D. R. Paul, and J. W. Barlow, J. Appl. Polym. Sci., 34, 537 (1987).

37. V. S. Shah, J. D. Keitz, D. R. Paul, and J. W. Barlow, J. Appl. Polym. Sci., 32, 3863 (1986).

38. T. K. Kwei, H. L. Frisch, W. Radigan, and S. Vogel, Macromolecules, 10, 157 (1977).

39. P. J. Flory, "Principles of Polymer Chemistry," Cornell University Press, Ithaca, N.Y., 1953, chapt. 12.

40. E. M. Woo, J. W. Barlow, and D. R. Paul, J. Appl. Polym. Sci., 29, 3837 (1984).

41. J. E. Harris, S. H. Goh, D. R. Paul, and J. W. Barlow, J. Appl. Polym. Sci., 27, 839 (1982).

42. M. Aubin and R. E. Prud'homme, Macromolecules, 13, 365 (1980). 